\title{
Design information orientation supporting system for
}

\section{user}

\section{Sinh Tien Vu ${ }^{1}$, Quyen Thi Vu ${ }^{1}{ }^{*}$, Duc Van Dang ${ }^{1}$, Huan Ngoc Le ${ }^{1}$, Minh Ngoc Pham ${ }^{1}$, Huong Thi Truong 2}

${ }^{1}$ Institute of Information Technology, Vietnam Academy of Science and Technology, Vietnam

${ }^{2}$ Falcuty of Mathematics, Thai Nguyen University of Education, Vietnam

\begin{abstract}
ARTICLE INFO
ABSTRACT

Article history:

Received $15^{\text {th }}$ Nov 2019

Revised $6^{\text {th }}$ Jan. 2020

Accepted 28 ${ }^{\text {th }}$ Feb. 2020

\section{Keywords:}

Internet Of Things (IoT),

Beacon, Bluetooth Low,

Energy,

Smart Supermarket.

The Bluetooth Low Energy (BLE) technology provides an effective means for indoor location and navigations by using Beacons - BLE devices that can transmit and receive signals over short distances. Device's information and goods' information that is linked to a Beacon can be archived by a mobile application, which is basically a Bluetooth - enabled application. In this paper, we present the design and development of an user - driven information IoT system based on the BLE technology. An experimental product, ESMarket, is a system that uses our self development Beacon - ESBeacon for smart supermarkets with the ESBeacons are placed in a small supermarket shelves and a Bluetooth enabled smartphone is used to install the digital supermarket application to communicate with these ESBeacons. The results show that when a smartphone appears within the ESBeacon range, it will display information related to the shelves on the screen.
\end{abstract}

Copyright (C) 2020 Hanoi University of Mining and Geology. All rights reserved.

${ }^{*}$ Corresponding author

E-mail:vtsinh.ictu@gmail.com

DOI: 10.46326/JMES.2020.61(1).05 


\title{
Tạp chí Khoa học Kỹ thuật Mỏ - Địa chất
}

Trang điện tử: http://tapchi.humg.edu.vn

\section{Thiết kế hệ thống IoT hỗ trợ định hướng thông tin cho người dùng}

\author{
Vũ Tiên Sinh ${ }^{1}$, Vũ Thị Quyên 1, ${ }^{*}$, Đặng Văn Đức ${ }^{1}$, Lê Ngọc Huân ${ }^{1}$, Phạm Ngọc Minh ${ }^{1}$, \\ Trương Thị Hương ${ }^{2}$
}

${ }^{1}$ Viện Công nghệ Thông tin, Viện Hàn lâm Khoa học và Công nghệ Việt Nam, Việt Nam

${ }^{2}$ Khoa Toán, Trường Đại học Sư phạm Thái Nguyên, Việt Nam

THÔNG TIN BÀI BÁO

TÓM TẮT

\section{Quá trình:}

Nhận bài 15/11/2019

Sửa xong 06/01/2020

Chấp nhận đăng 28/02/2020

\section{Tù̀ khóa:}

Internet Of Things (IoT),

Beacon, Bluetooth Low,

Energy,

Smart Supermarket.
Giao thức vô tuyến năng lượng thấp BLE (Bluetooth Low Energy) cung cấp một phương tiện hiệu quả để định vị và điều hướng trong nhà bằng cách sư dụng Beacons - các thiết bị BLE có thể phát và nhận tín hiệu trong một khoảng cách ngắn. Trong bài báo này, nhóm tác giả trình bày việc xây dựng và phát triển hệ thống IoT hỗ trợ định hướng thông tin người dùng trên nền tảng công nghệ BLE. Sản phẩm thử nghiệm là ESMarket: một hệ thống với sự hỗ trợ của các ESBeacon cho siêu thị thông minh với các ESBeacon được đặt tại các kệ hàng trong siêu thị nhỏ và điện thoại thông minh hố trợ Bluetooth sử dụng để cài đặt ứng dưng siêu thị số để giao tiếp với các beacon. Kết quả cho thấy khi điện thoại thông minh xuất hiện trong phạm vi của ESBeacon, nó sẽ hiển thị thông tin liên quan đến kệ hàng trên màn hình điện thoai.

C 2020 Trường Đại học Mỏ - Địa chất. Tất cả các quyền được bảo đảm.

\section{Mở đầu}

Hệ thống IoT hỗ trợ định hướng thông tin cho người dùng là một hệ thống trong mô hình tiếp thị và quảng cáo kết nối. Hệ thống này nhằm đưa ra một giải pháp nhằm thay đổi cách tiếp cận của doanh nghiệp với khách hàng của mình cũng như trong các công tác phân tích thói quen tiêu dùng, tối ưu hoá sự tương tác, ... Hệ thống này cho phép doanh nghiệp thay đổi hoàn toàn góc nhìn đối với khách hàng và phát triển những thông điệp riêng

\footnotetext{
*Tác giả liên hệ

E - mail: vtsinh.ictu@gmail.com

DOI: 10.46326/JMES.2020.61(1).05
}

với các thiết bị đem lại lợi ích lớn nhất cho họ. Trong khi đó, về phía người dùng, việc thanh toán dễ dàng, tiếp cận thông tin nhanh chóng, ... cũng là tiền đề cho mức tăng lợi nhuận không nhỏ.

Có nhiều công nghệ có thể được sử dụng trong các hệ thống IoT như RFID, EPC, NFC, Bluetooth, Z - Ware, hay Wifi. Trong đó, BLE (Bluetooth Low Energy) đang trở nên phổ biến như một cách giao tiếp không dây thuận tiện do yêu cầu năng lượng thấp và đặc tính không tốn kém so với Bluetooth cổ điển (Bluetooth Classic) (Mohamad Omar Al Kalaa, et al, 2016) (Monika Adarsh, 2018). Điều này khiến cho BLE trở nên đặc biệt, các doanh nghiệp có thể sử dụng chỉ bốn quả pin để cấp nguồn cho các thiết bị Bluetooth trong vài tháng hoặc nhiều năm. Trong khi Bluetooth cổ điển phù 
hợp cho các ứng dụng yêu cầu truyền dữ liệu liên tục, ví dụ như tai nghe hay chuyển tệp tin giữa các thiết bị, BLE lại phù hợp với các ứng dụng cho việc truyền dữ liệu định kỳ, do đó làm giảm giảm đáng kể tiêu hao pin (Monika Adarsh, 2018). Với lợi thế đó, nhóm tác giả đã ứng dụng công nghệ BLE để thiết kế chế tạo thành công thiết bị ESBeacon cho một hệ thống IoT định hướng thông tin cho người dùng.

\section{Cơ sở công nghệ Beacon}

\subsection{Công nghệ Beacon là gì?}

Beacon (điểm hiệu) là công nghệ đang được nhiều công ty ở mọi lĩnh vực trên thế giới triển khai như là những điểm thu sóng Bluetooth để báo cho điện thoại thông minh chính xác địa điểm của chúng ở đâu, qua đó một ứng dụng có thể hành xử tương ứng với vị trí cụ thể đó.

Beacon sử dụng công nghệ BLE (Bluetooth Low Energy), còn được gọi là Bluetooth Smart. BLE sử dụng giao thức khác Classic Bluetooth và không tương thích ngược. Đây là một công nghệ có sẵn trên iPad và iPhone từ đời 2010 (và Mac từ đời 2012), và trong các thiết bị Android cao cấp từ đời 2013. Chúng không cần có bộ đọc chuyên dụng, chỉ cần chiếc điện thoại thông minh là đủ. Đó là lý do tại sao Beacon rất tiềm năng, sẽ sớm trở thành công cư xác định vị trí trong mơ mà RFID từng ao ước có được. Một đặc điểm nổi trội chính của Beacon so với GPS đó là: Beacon không gửi dữ liệu vị trí địa lý nhưng gửi dữ liệu nhận diện thiết bị. Công việc còn lại là nhiệm vụ của các thành phần còn lại của hệ thống được xây dựng sẽ làm gì khi nhận diện được có một Beacon gần đó.

Đặc điểm:

- Tiết kiệm năng lượng, cho phép Beacon truyền thông tin liên tục lên đến 2 - 3 năm chỉ với một viên pin nhỏ.

- Khoảng cách truyền BLE cũng lên đến 100 m như Classic Bluetooth.

- BLE sử dụng cùng băng tần $2.4 \mathrm{GHz}$

Sơ đồ khối thiết bị định danh ESBeacon được thể hiện trong Hình 1.

Chức năng:

- Chíp nRF52832 của hãng Nodrdic được tích hợp trong Module Bluetooth E73 - 2G4M04S.

- Khối nguồn: Với chức năng cung cấp nguồn cho mạch. Trong khuôn khổ bài báo này, chúng tôi thiết kế mạch thử nghiệm với 2 lựa chọn: nguồn pin và nguồn điện, tùy vào ứng dụng của hệ thống.

- Nút ấn điều khiển chỉ giữ chức năng bật tắt nguồn hoạt động.

- Đèn tín hiệu với chức năng thông báo tình trạng hoạt động của mạch.

- Anten: Để tăng khả năng truyền xa cho mạch.

\subsection{Giao thức truyền dũ̃ liệu Beacon}

Giao thức truyền dữ liệu và kết nối với phần mềm trên điện thoại (Hình 2, 3).

Mỗi ESBeacon có các thông số cơ bản, đó là, chuẩn định dạng iBeacon có ba giá trị là UUID,

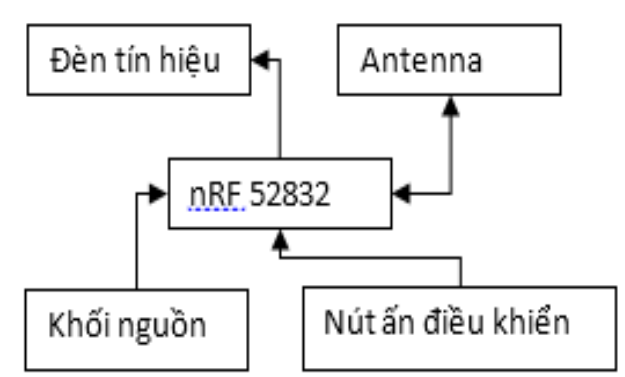

Hình 1. Sơ đồ khối của thiết bị định danh ESBeacon sử dụng công nghệ Bluetooth.

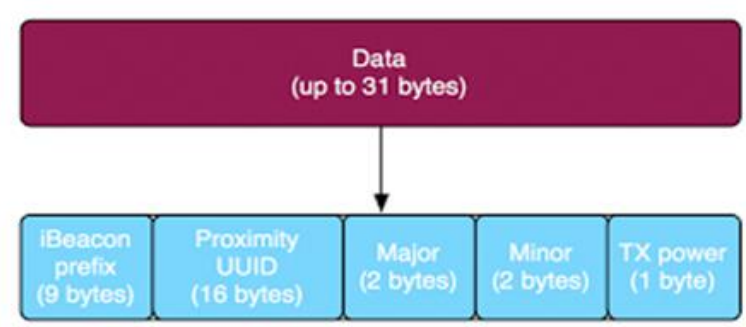

Hình 2. Packet Data Unit (PDU) của iBeacon.

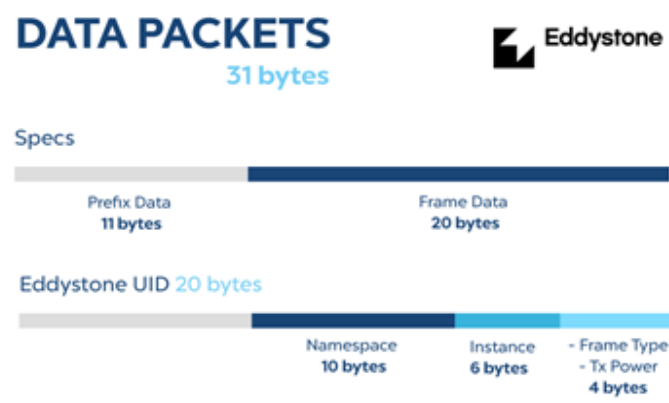

Eddystone URL 6-20 bytes

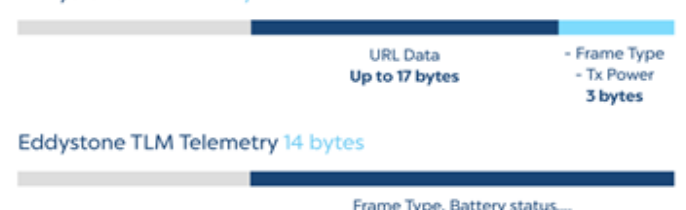

Frame Type, Battery status,..

Hình 3. Packet Data Unit (PDU) của EddyStone. 
Major ID và Minor ID, chuẩn định dạng EddyStone bao gồm Namespace, Instance đối với EddyStone UID. Các giá trị này được xác định bởi ứng dụng di động để kết xuất dữ liệu đầu ra tương ứng với từng ESBeacon.

\subsection{Phương pháp tính khoảng cách của BLE}

Các mô hình BLE sử dụng chỉ số cường độ tín hiệu thu RSSI (Received Signal Strength Indicator) trong tính toán khoảng cách hay vị trí như tham số cơ bản. Việc tính khoảng cách được dựa trên các mô hình tham số. Nhóm tác giả thử nghiệm hai mô hình tham số đó là: log shadowing và mô hình của bộ thư viện Android Beacon Library.

Một tham số tham chiếu RSSIre sử dụng trong mô hình tính toán được định nghĩa, là giá trị trung bình của RSSI khi khoảng cách từ thiết bị BLE đến thiết bị đo là $1 \mathrm{~m}$.

- Mô hình log shadowing (LogR) sử dụng công thức tính khoảng cách sau (Rappaport, 1996)

$$
d=10^{\frac{R S S I-R S S I_{r e}}{-10 n}}
$$

Trong đó: $\mathrm{n}$ là PLE (path loss exponent), $n=2$ khi thực hiện tính toán ở trong khoảng không gian trống.

- Mô hình Android Beacon Library (https:// altbeacon.github.io/android - beacon - library/ distance - calculations.html) sử dụng công thức:

$$
d=A \cdot\left(\frac{R S S I}{R S S I}\right)^{B}+C
$$

Trong đó: $A, B, C$ là các hằng số được tính bằng cách sử dụng phương pháp hồi quy lũy thừa (power regression) trên tập dữ liệu đầu vào được thu thập từ trước. Các bước thực hiện như sau:

Sử dụng 1 điện thoại iPhone để đo RSSI ở khoảng cách $1 \mathrm{~m}$ làm giá trị tham chiếu.

Lập bảng đo RSSI trên thiết bị cài đặt thử nghiệm ở 20 điểm có khoảng cách khác nhau: $0.25,0.5,1,2,3,4,5,6,7,8,9,10,12,14,16,18,20$, $25,30,40$.

Lập bảng tham số đầu vào cho phép tính hồi quy $y=A^{*} x^{B}$, biến độc lập là giá trị 20 điểm đo bằng mét, biến phụ thuộc là tỉ lệ giữa RSSI đo được trên thiết bị thử nghiệm và thiết bị tham chiếu.

Sử dụng công cụ để chạy hàm hồi quy sẽ thu được kết quả $A$ và $B$.

Tính khoảng cách dự đoán bằng sử dụng $\mathrm{A}, \mathrm{B}$ thu được ở trên vào công thức:

$$
y=A^{*} X^{B}
$$

Tính $C$ bằng cách lấy khoảng cách đo thực tế trừ đi khoảng cách đự đoán.

Tính lại khoảng cách dự đoán bằng cách sử dụng $\mathrm{C}$ ở bước trên vào công thức ban đầu.

\section{Phân tích thiết kế hệ thống}

\subsection{Xây dựng mô hình hệ thống IoT định hướng thông tin cho người dùng}

Một ứng dụng siêu thị số được tạo ra để hiển thị thông tin về các chương trình giảm giá, khuyến

\begin{tabular}{|c|c|c|c|c|c|c|c|c|c|c|c|c|c|c|c|c|c|c|}
\hline \\
\hline \multicolumn{19}{|c|}{ iPhone 1m RSSI } \\
\hline \multicolumn{19}{|c|}{-62.68} \\
\hline & & \multicolumn{2}{|c|}{ Step 1: Caclulate Ratio } & \multicolumn{8}{|c|}{ Step 2: Dữ liệu hồi qı Step 3: Chạy thuật toán hi Step 4. Tính kc theo A \& B } & & \multicolumn{2}{|c|}{ Step 5. Tính C } & \multicolumn{4}{|c|}{ Step 8. Tính k/c theo A, B \& C } \\
\hline TABLE 2 & & & & TABLE 3 & & \multicolumn{3}{|c|}{ TABLE 4} & \multicolumn{2}{|c|}{ TABLE 5} & & & \multicolumn{2}{|c|}{ TABLE 6} & \multicolumn{2}{|c|}{ TABLE 7} & & \\
\hline \multicolumn{2}{|l|}{ Thu thập dữ liệu } & & & Các tham & & & ng trang $h$ & http://unw. & v. công thú & nức $d=A^{*}(r / t)^{\wedge} B$ & & & \multicolumn{2}{|c|}{ Sai số ở k/c $1 \mathrm{~m}$} & \multicolumn{4}{|c|}{ Công thức $\mathrm{d}=\mathrm{A}^{*}(\mathrm{r} / \mathrm{t})^{\wedge} \mathrm{B}+\mathrm{C}$} \\
\hline Distance $(\mathrm{m})$ & RSSI & I Ratio (RSSVlphon & $1 \mathrm{e} R \mathrm{~m}$ & S Phụ thuộc & Độc lập & A & 4.295 & & RSSI & Ratio & K/c thực & $\mathrm{K} / \mathrm{c}$ tính toán & Calc & ation of $\mathrm{C}$ & RSSI & Ratio & Actual D & Predicte \\
\hline 0.25 & -41 & 0.654116146 & & 0.6541 & 0.25 & B & 6.17 & & -41 & 0.6541161 & 0.25 & 0.313 & C & 0.0598 & -41 & 0.6541 & 0.25 & 0.373 \\
\hline 0.5 & -43 & 0.68602425 & & 0.686 & 0.5 & & & & -43 & 0.6860243 & 0.5 & 0.42 & & & -43 & 0.686 & 0.5 & 0.48 \\
\hline 1 & -49 & 0.781748564 & & 0.7817 & 1 & & & & -49 & 0.7817486 & 1 & 0.9402 & & & -49 & 0.7817 & 1 & 1 \\
\hline 2 & -65 & 1.037013401 & & 1.037 & 2 & & & & -65 & 1.0370134 & 2 & 5.3752 & & & -65 & 1.037 & 2 & 5.435 \\
\hline 3 & -58 & 0.925335035 & & 0.9253 & 3 & & & & -58 & 0.925335 & 3 & 2.6611 & & & -58 & 0.9253 & 3 & 2.721 \\
\hline 4 & -57 & 0.909380983 & & 0.9094 & 4 & & & & -57 & 0.909381 & 4 & 2.3904 & & & -57 & 0.9094 & 4 & 2.45 \\
\hline 5 & -67 & 1.068921506 & & 1.0689 & 5 & & & & -67 & 1.0689215 & 5 & 6.4804 & & & -67 & 1.0689 & 5 & 6.54 \\
\hline 6 & -67 & 1.068921506 & & 1.0689 & 6 & & & & -67 & 1.0689215 & 6 & 6.4804 & & & -67 & 1.0689 & 6 & 6.54 \\
\hline 7 & -77 & 1.228462029 & & 1.2285 & 7 & & & & -77 & 1.228462 & 7 & 15.289 & & & -77 & 1.2285 & 7 & 15.35 \\
\hline 8 & -70 & 1.116783663 & & 1.1168 & 8 & & & & -70 & 1.1167837 & 8 & 8.4914 & & & -70 & 1.1168 & 8 & 8.551 \\
\hline 9 & -69 & 1.100829611 & & 1.1008 & 9 & & & & -69 & 1.1008296 & 9 & 7.77 & & & -69 & 1.1008 & 9 & 7.83 \\
\hline 10 & -75 & 1.196553925 & & 1.1966 & 10 & & & & -75 & 1.1965539 & 10 & 12.997 & & & -75 & 1.1966 & 10 & 13.06 \\
\hline 12 & -72 & 1.148691768 & & 1.1487 & 12 & & & & -72 & 1.1486918 & 12 & 10.103 & & & -72 & 1.1487 & 12 & 10.16 \\
\hline 14 & -72 & 1.148691768 & & 1.1487 & 14 & & & & -72 & 1.1486918 & 14 & 10.103 & & & -72 & 1.1487 & 14 & 10.16 \\
\hline 16 & -78 & 1.244416082 & & 1.2444 & 16 & & & & -78 & 1.2444161 & 16 & 16.556 & & & -78 & 1.2444 & 16 & 16.62 \\
\hline 18 & -83 & 1.324186343 & & 1.3242 & 18 & & & & -83 & 1.3241863 & 18 & 24.291 & & & -83 & 1.3242 & 18 & 24.35 \\
\hline 20 & -81 & 1.292278239 & & 1.2923 & 20 & & & & -81 & 1.2922782 & 20 & 20.897 & & & -81 & 1.2923 & 20 & 20.96 \\
\hline 25 & -81 & 1.292278239 & & 1.2923 & 25 & & & & -81 & 1.2922782 & 25 & 20.897 & & & -81 & 1.2923 & 25 & 20.96 \\
\hline 30 & -75 & 1.196553925 & & 1.1966 & 30 & & & & -75 & 1.1965539 & 30 & 12.997 & & & -75 & 1.1966 & 30 & 13.06 \\
\hline 40 & -83 & 1.324186343 & & 1.3242 & 40 & & & & -83 & 1.3241863 & 40 & 24.291 & & & -83 & 1.3242 & 40 & 24.35 \\
\hline
\end{tabular}
mại, thông tin về các sản phẩm trên kệ hàng. Hình 5 là kiến trúc hệ thống của giải pháp đề xuất.

Hình 4. Chuẩn bị dũ liệu và các bước tính toán. 


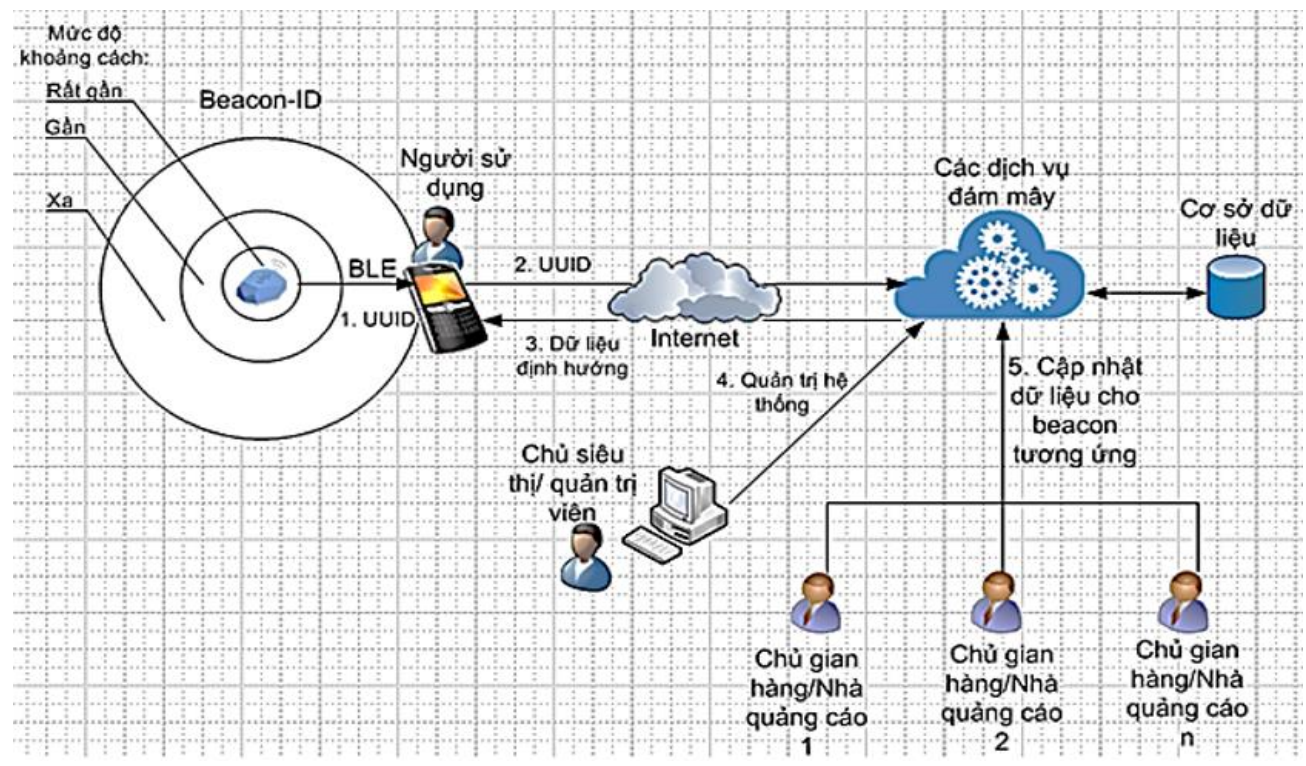

Hình 5. Kiến trúc hệ thống.

Hệ thống này là một bộ sản phẩm bao gồm các thành phần chính:

- Thiết bị định danh ESBeacon có hình dạng nhỏ gọn sử dụng công nghệ BLE kết nối với điện thoại thông minh, máy tính bảng.

- Phần mềm server trên máy chủ đám mây lưu trữ dữ liệu hành vi, đánh giá nhu cầu người sử dụng, tiếp nhận và trả lời truy vấn thông tin định hướng tới người dùng.

- Phần mềm client trên điện thoại thông minh, máy tính bảng phát hiện và giao tiếp với thiết bị định danh ESBeacon.

\subsection{Thiết kế phần cứng thiết bị ESBeacon}

Đối với ESBeacon có 3 vấn đề quan trọng cần đề cập:

- Khoảng cách truyền của mạch: trên thực tế thử nghiệm, mạch có thể truyền xa $40 \mathrm{~m}$ (không vật cản) khi không có anten. Khoảng cách truyền có thể lên tới 100 m, với điều kiện thử nghiệm thoáng và không bị nhiễu, có anten. Do đó anten sẽ được sử dụng tùy vào yêu cầu sử dụng của từng ưng dụng và đơn vị hợp tác.

- Năng lượng pin cung cấp cho mạch hoạt động: đối với chế độ chạy bằng điện, thì nguồn lúc nào cũng đủ cung cấp cho mạch hoạt động ở chế độ ổn định nhất (Hình 6). Tuy nhiên đối với nguồn pin, vấn đề đặt ra là thời gian hoạt động của pin cung cấp cho mạch hoạt động là bao lâu? Mạch có chạy ổn định không?
+ Đối với mạch thử nghiệm phiên bản đầu tiên này, nguồn pin sẽ cung cấp điện cho Module Bluetooth E73 - 2G4M04S là chính và cung cấp điện cho 1 đèn báo tín hiệu mạch đang hoạt động (năng lượng cung cấp cho đèn là không đáng kể).

Ta có:

- Nguồn Pin CR2477 (3V, 1000 mAh).

- Module E73 - 2G4M04 với công suất truyền lớn nhất là $2,5 \mathrm{~mW}=0.0025 \mathrm{~W}$.

Vậy dòng truyền của module là: $0.0025 / 3=$ $0.0008 \mathrm{~A}$; thời gian hoạt động của pin cung cấp cho mạch là: $1 / 0.0008=1250$ giờ.

Đây là thời gian mà mạch có thể truyền dữ liệu liên tục (kết nối truyền dữ liệu liên tục với app trên điện thoại). Giả sử mạch làm việc liên tục 8h/ngày thì mạch sẽ làm việc được: 1250/8 = 156 ngày $\sim 5$ tháng.

Trong khi đó, dòng điện tiêu thụ khi ngủ của mạch chỉ là: $2^{*} 10^{-4} \mathrm{~mA}$. Thì nếu trong trạng thái ngủ liên tục thì mạch có thể hoạt động trong thời gian là: $\frac{1000}{2.10^{-4}}=5000000$

Có thể nói rằng, năng lượng điện tiêu thụ của mạch khi ngủ là không đáng kể.

Do đó, với các ứng dụng khác nhau của mạch thì thời gian hoạt động của mạch mà pin có thể cung cấp là khác nhau. Thời gian từ 5 tháng tới 1 năm. (Siêu thị làm việc liên tục là 5 tháng, còn với các ứng dụng như viện bảo tàng thì sẽ lâu hơn, ...).

+ Trong quá trình chạy thử nghiệm 6 tháng thì thiết bị ESBeacon vẫn chạy ổn định, không xảy ra 
vấn đề mất kết nối với App điện thoại của người dùng.

\subsection{Thiết kế phần mềm trên máy chủ}

Ứng dụng của máy chủ được cài đặt trên đám mây được sử dụng để quản lý các ESBeacon. Nó cho phép chủ siêu thị hay chủ gian hàng có thể truy cập để quản lý các ESBeacon cũng như nội dung sẽ được gửi tới màn hình điện thoại của người sử dụng tương ứng với từng ESBeacon (Hình 7).

\subsection{Thiết kế phần mềm client trên thiết bị di động}

Phần mềm Client trên điện thoại thông minh, máy tính bảng phát hiện và giao tiếp với thiết bị định danh ESBeacon, từ đó đưa ra dữ liệu định hướng người dùng tương ứng. Chức năng của phầm mềm được biểu diễn bằng sơ đồ chức năng (Hình 8, 9).

\section{Tích họ̣p và thử nghiệm}

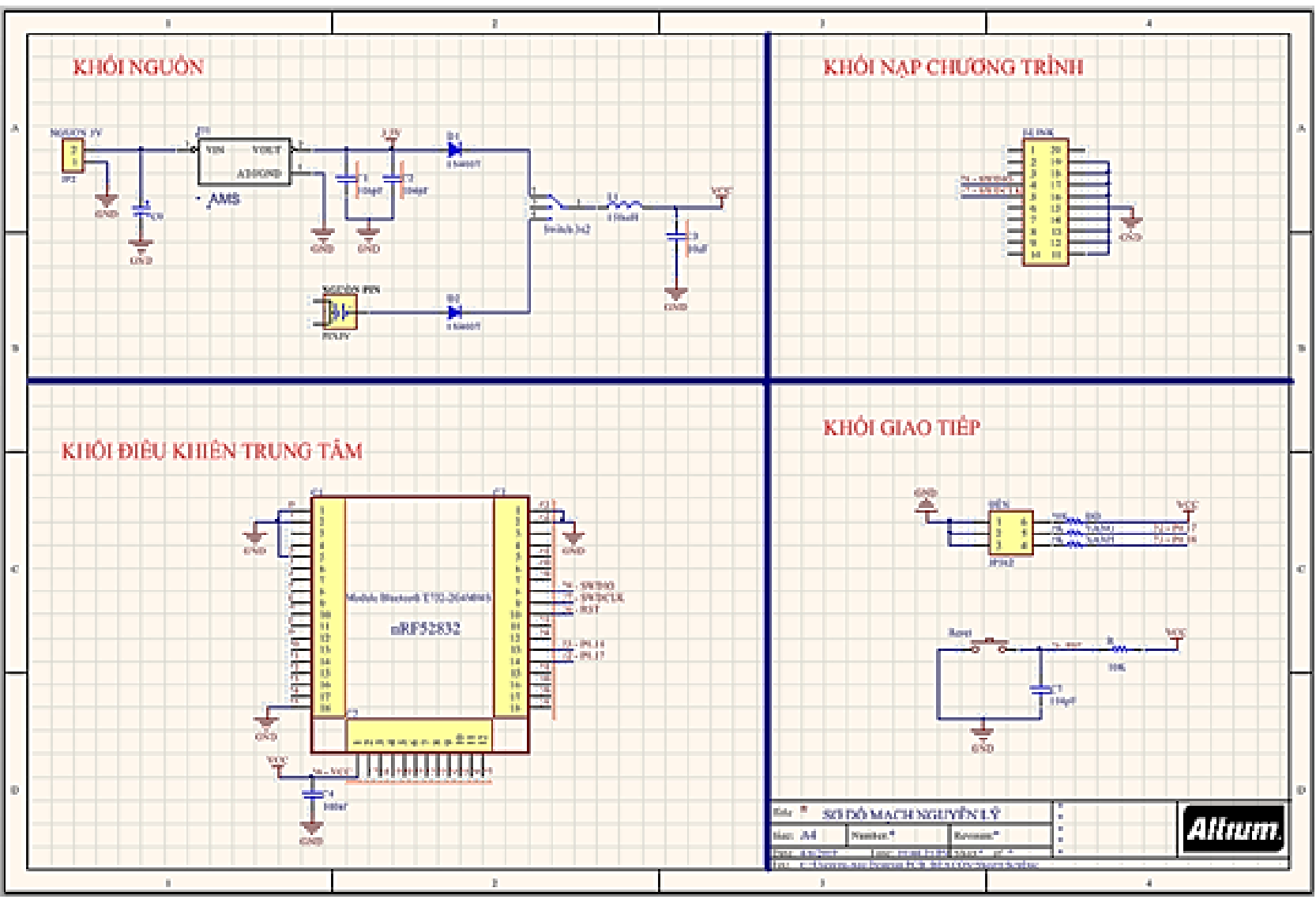

Hình 6. Sơ đồ mạch nguyên lý thiết bị ESBeacon.

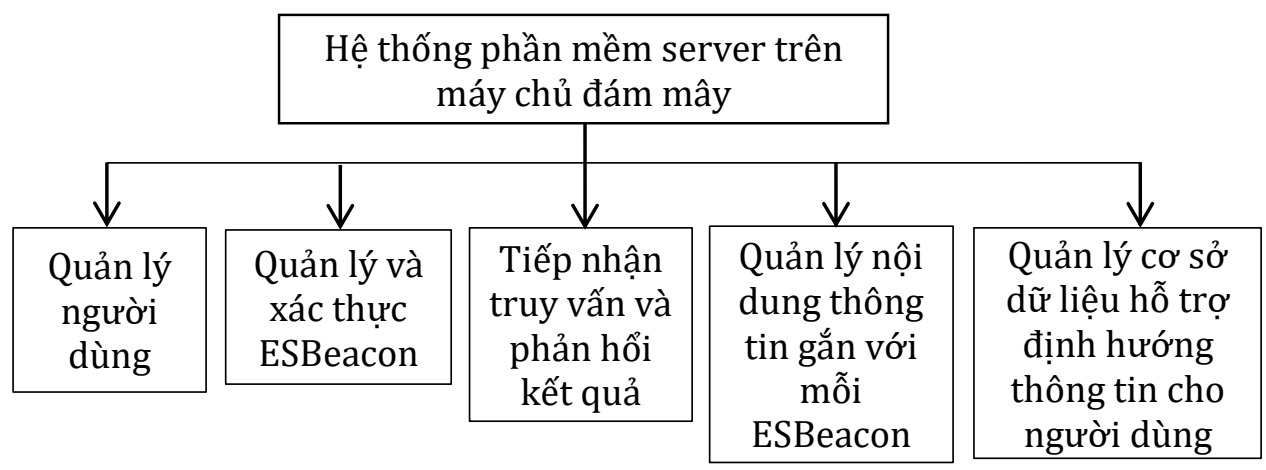

Hình 7. Sơ đồ chức năng của ứng dụng phía máy chủ. 


\subsection{Tích hợp và chế tạo thiết bị ESBeacon}

Qua quá trình nghiên cứu và thử nghiệm tại phòng thí nghiệm, nhóm tác giả đã đưa ra sản phẩm ESBeacon (Hình 10, Bảng 1)

\subsection{Phần mềm úng dụng ESMarket}

Siêu thị Traumerei, số 49 Quang Trung, Hà Đông, Hà Nội được chọn để thử nghiệm ESMarket vì đây là một siêu thị với số lượng mặt hàng và kệ hàng vừa đủ để thử nghiệm. Nó cũng được chọn để kiểm tra hoạt động của ESMarket ở những khu vực đông người vì tín hiệu của beacon sẽ bị giao thoa và hấp thụ.

Các ESBeacon được gắn trên các kệ hàng cách nhau 1 đến 5 mét trong siêu thị. Sử dụng ESMarket, người mua sắm có thể tìm thấy những kệ hàng một cách dễ dàng, kèm theo đó là các thông tin liên quan đến kệ hàng cũng được hiển thị ngay trên màn hình điện thoại thông minh của họ. Hệ thống đề xuất được thử nghiệm bởi các nhân viên của siêu thị và đội ngũ phát triển hệ thống. Một ESBeacon được đặt tại cưa ra vào của siêu thị, hai ESBeacon được đặt tại các kệ hàng bao gồm các thông tin cơ bản như trong Bảng 2 .

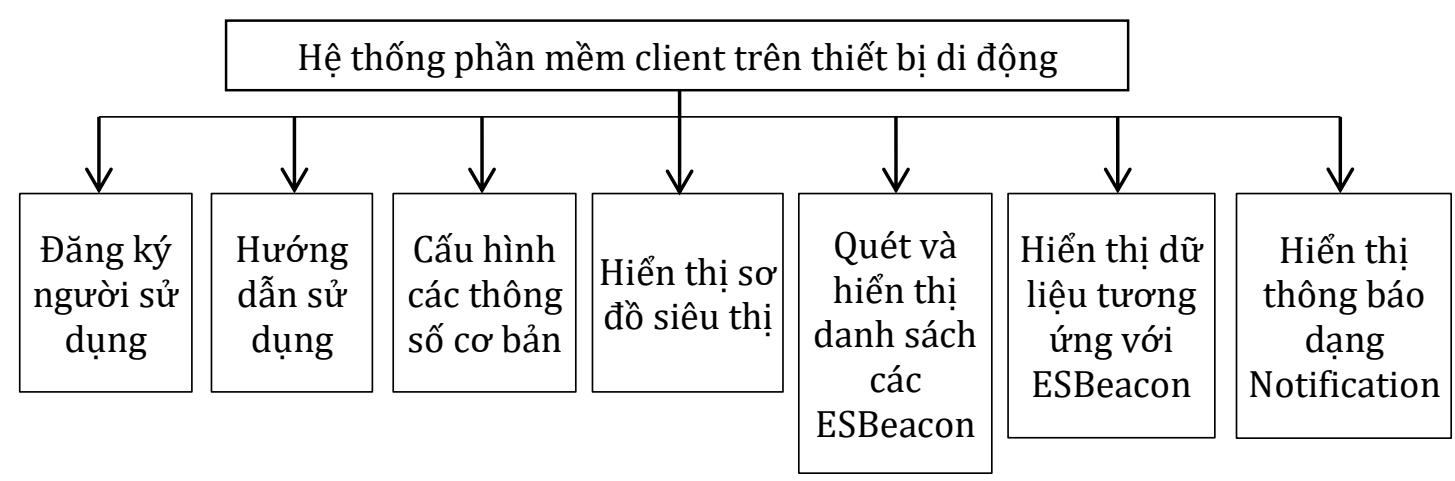

Hình 8. Sơ đồ chức năng phần mềm client trên thiết bị di động.

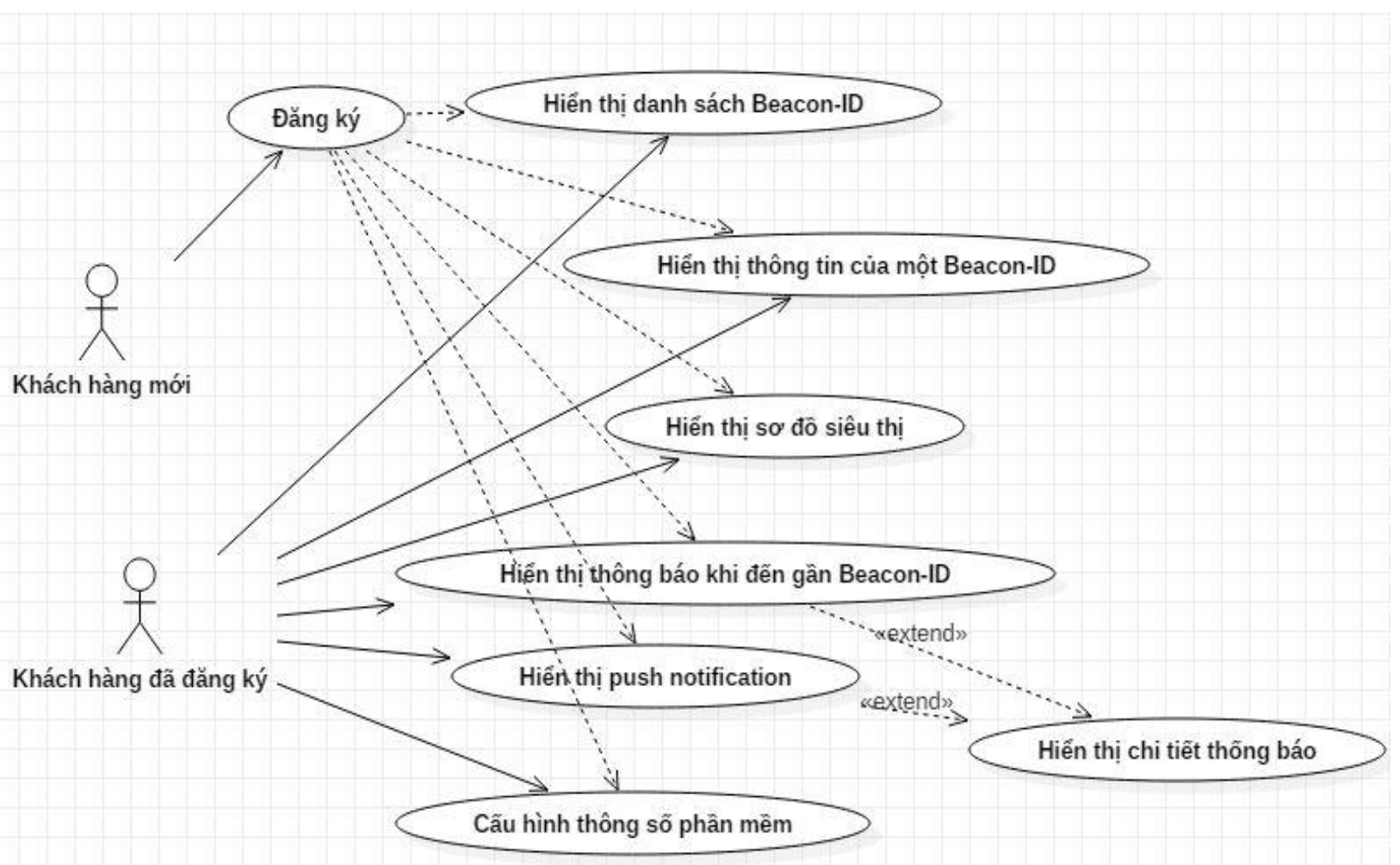

Hình 9. Biểu đồ usecase tổng quát của phần mềm client. 


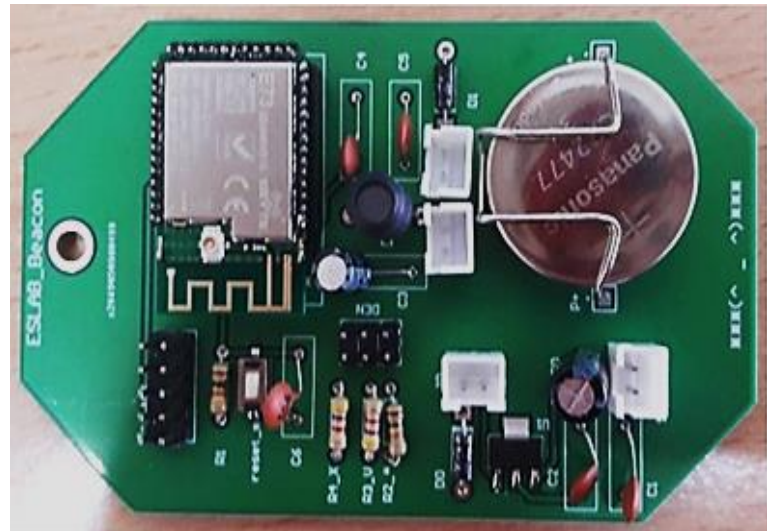

Hình 10. Sản phẩm hoàn thiện.

Bảng 1. So sánh với "Thiết bị iBeacon Estimote" đang bán trên thị trường Việt Nam.

\begin{tabular}{|c|c|c|}
\hline MCU & $\begin{array}{c}\text { nRF52832 } \\
\text { NODRDIC }\end{array}$ & nRF52832 NODRDIC \\
\hline $\begin{array}{c}\text { Chuẩn kết } \\
\text { nối }\end{array}$ & $\begin{array}{c}\text { Bluetooth® } 4.2 \mathrm{LE} \\
\text { standard }\end{array}$ & $\begin{array}{c}\text { Hỗ trợ các chuẩn BLE } \\
\text { 4.2 và BLE 5.0 mới } \\
\text { nhất bây giờ. }\end{array}$ \\
\hline Radio & $2.4 \mathrm{GHz}$ transceiver & $2.4 \mathrm{GHz}$ transceiver \\
\hline Dải tần số & $\begin{array}{c}2400 \mathrm{MHz} \text { to } \\
2483.5 \mathrm{MHz}\end{array}$ & $2379 \sim 2496 \mathrm{MHz}$ \\
\hline Độnhạy & $-96 \mathrm{dBm}$ & $-96 \mathrm{dBm}$ \\
\hline Pin & CR2477 (USA) & CR2477 (indonesia) \\
\hline $\begin{array}{c}\text { Khoảng } \\
\text { cách truyền }\end{array}$ & $100 \mathrm{~m}$ & $100 \mathrm{~m}$ \\
\hline
\end{tabular}

Bảng 2. ESBeacon và thông tin liên quan.

\begin{tabular}{|c|c|c|c|c|}
\hline Beacon & Text & Image & Video & Link \\
\hline $\begin{array}{c}\text { Cửa ra } \\
\text { vào }\end{array}$ & $\begin{array}{l}\text { Xin chào } \\
\text { quý khách } \\
\text { đến với } \\
\text { siêu thị } \\
\text { Traumerei }\end{array}$ & $\begin{array}{c}\text { Ảnh sơ đồ } \\
\text { siêu thị }\end{array}$ & \begin{tabular}{|} 
Link video \\
giới thiệu \\
siêu thị
\end{tabular} & $\begin{array}{c}\text { Link tới } \\
\text { website } \\
\text { của siêu } \\
\text { thị }\end{array}$ \\
\hline $\begin{array}{c}\text { Sức } \\
\text { khỏe } \\
\text { và làm } \\
\text { đẹp }\end{array}$ & $\begin{array}{l}\text { Gian hàng } \\
\text { sức khỏe } \\
\text { và làm đẹp }\end{array}$ & $\begin{array}{c}\text { Ảnh } \\
\text { chương } \\
\text { trình } \\
\text { khuyến } \\
\text { mại của } \\
\text { gian hàng } \\
\text { sức khỏe } \\
\text { và sắc đẹp }\end{array}$ & $\begin{array}{l}\text { Link video } \\
\text { giới thiệu } \\
\text { các sản } \\
\text { phẩm } \\
\text { dành cho } \\
\text { sức khỏe } \\
\text { và làm đẹp }\end{array}$ & $\begin{array}{l}\text { Link chi } \\
\text { tiết các } \\
\text { sản phẩm } \\
\text { trên trang } \\
\text { web của } \\
\text { siêu thị }\end{array}$ \\
\hline Rượu & $\begin{array}{l}\text { Gian hàng } \\
\text { rượu }\end{array}$ & $\begin{array}{c}\text { Ảnh } \\
\text { chương } \\
\text { trình } \\
\text { khuyến } \\
\text { mại của } \\
\text { gian hàng } \\
\text { rưou }\end{array}$ & \begin{tabular}{|} 
Link video \\
giới thiệu \\
các sản \\
phẩm của \\
gian hàng \\
rượu
\end{tabular} & $\begin{array}{c}\text { Link chi } \\
\text { tiết các } \\
\text { sản phẩm } \\
\text { trên trang } \\
\text { web của } \\
\text { siêu thị }\end{array}$ \\
\hline
\end{tabular}

Khi người sử dụng đi ngang qua siêu thị hoặc bắt đầu đi qua cửa ra vào của siêu thị, một thông báo sẽ xuất hiện trên điện thoại thông minh của người sử dụng kèm theo đó là sơ đồ các kệ hàng trong siêu thị, giúp cho người sử dụng có thể định vị các kệ hàng một cách nhanh chóng. Khi người

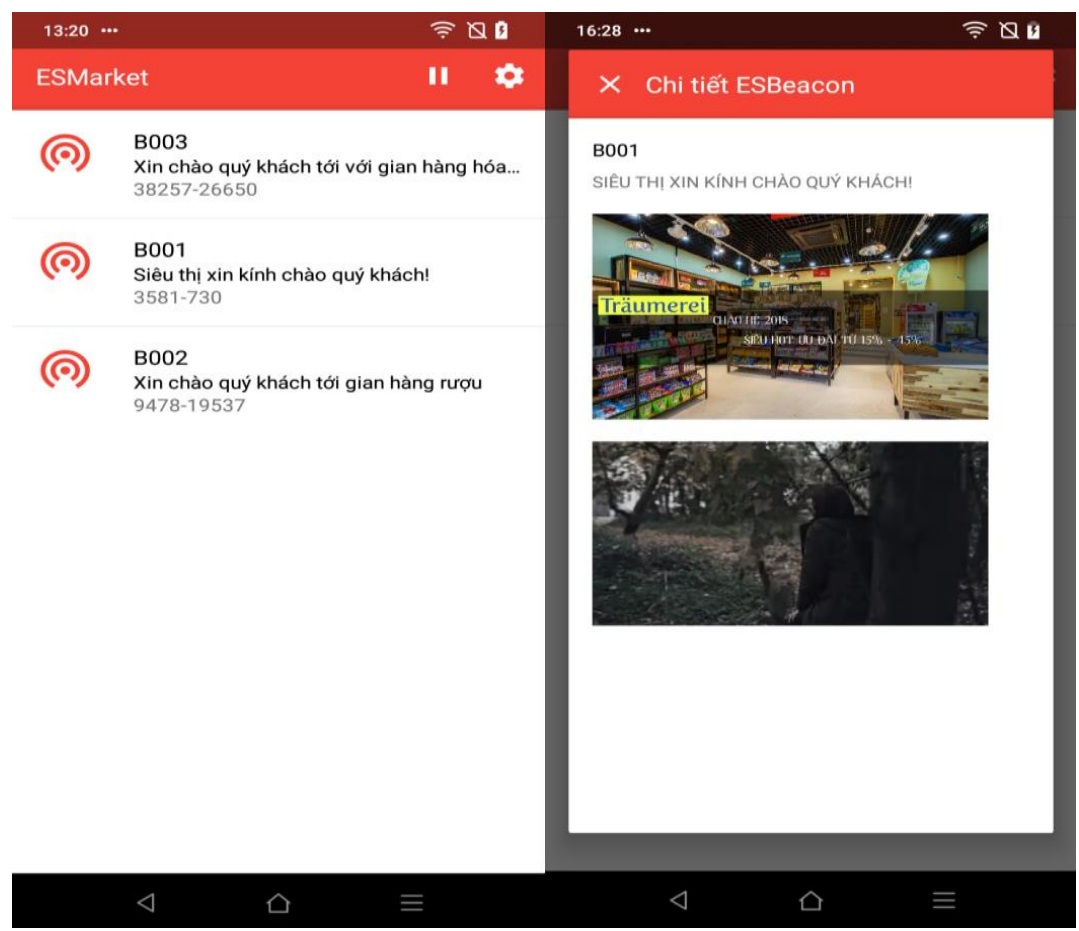

Hình 11. Màn hình hiển thị thông tin trên điện thoại thông minh của người sử dụng. 
sử dụng tiếp cận với các ESBeacon trên kệ hàng cụ thể, thông tin liên quan đến kệ hàng sẽ được hiển thị trên điện thoại thông minh của người sử dụng. Lưu đồ của ứng dụng ESMarket được hiển thị trong Hình 12. Đầu tiên, người dùng cần cài đặt ứng dụng siêu thị số ESMarket. Sau đó, ứng dụng sẽ kiểm tra xem người dùng cài đặt ứng dụng lần đầu hay không để hiển thị màn hình đăng ký sử dụng. Tiếp đến, ứng dụng sẽ kiểm tra xem Bluetooth trên thiết bị của người sử dụng đang bật hay tắt. Nếu bật Bluetooth người sử dụng sẽ nhận được thông tin tùy thuộc vào vị trí đứng hiện tại. Nếu người sử dụng ở gần ESBeacon "Cửa ra vào" thì ứng dụng sẽ hiển thị lời chào hình ảnh sơ đồ siêu thị; nếu người sử dụng ở gần ESBeacon "Sức khỏe \& làm đẹp" thì ứng dụng sẽ hiển thị thông tin cơ bản về kệ hàng, thông tin khuyến mại, giảm giá, video giới thiệu về các sản phẩm, liên kết tới chi tiết các sản phẩm. Tương tự nếu người sử dụng ở gần ESBeacon "Rượu" thì ứng dụng sẽ hiển thị thông tin cơ bản của kệ hàng "Rượu". Sau đó, nếu người dùng đi đến ESBeacon khác thì ứng dụng sẽ hiển thị thông tin của kệ hàng liên quan đến ESBeacon đó. Nếu người sử dụng rời khỏi siêu thị hoặc không muốn có thêm thông tin nào về kệ hàng thì họ có thể tắt Bluetooth. Bằng cách này,

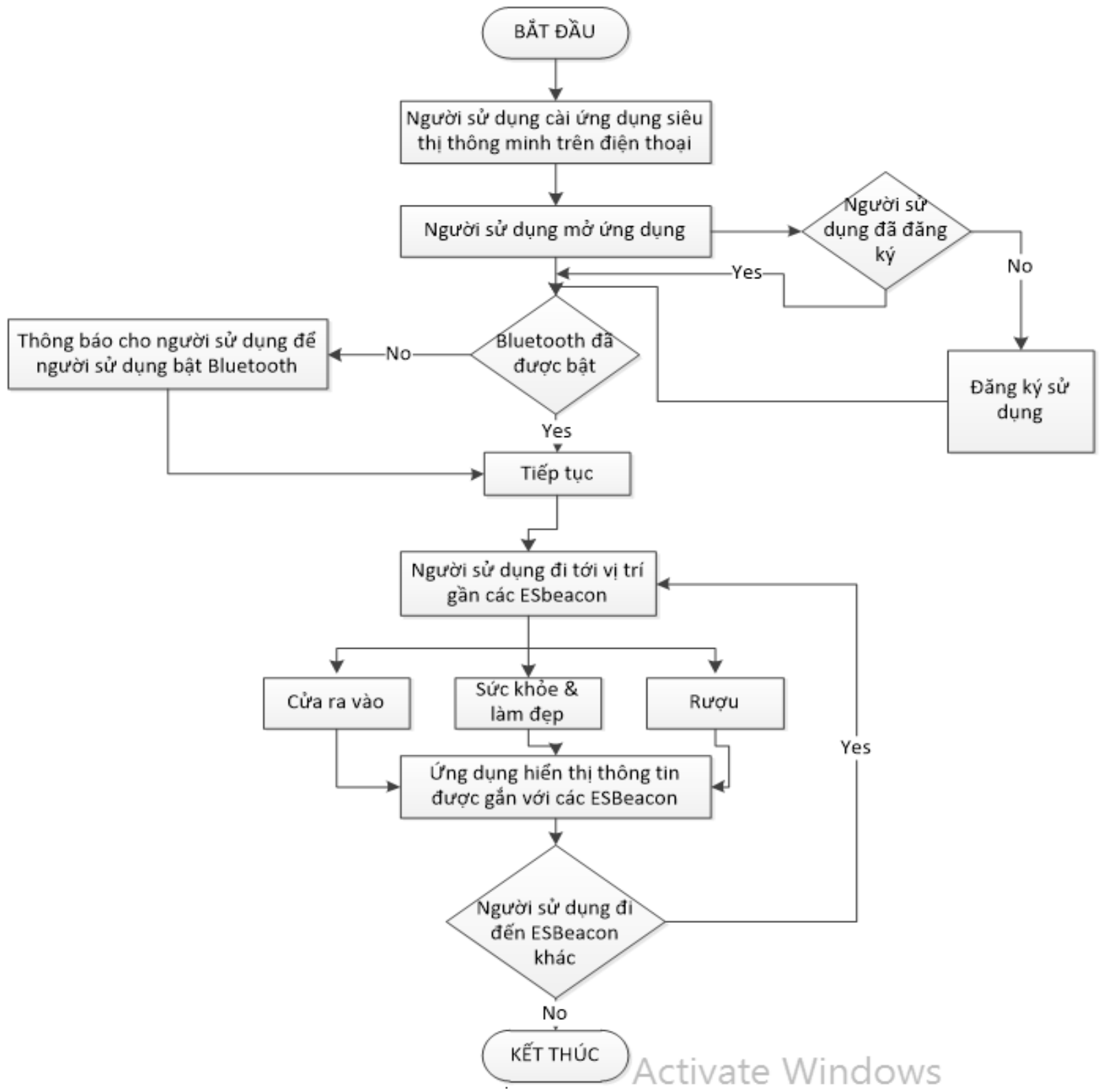

Hình 12. Lưu đồ úng dụng ESMarket. 
người dùng có thể có được các thông tin một cách nhanh chóng mà không cần phải đi tới gần kệ hàng và tìm kiếm từng sản phẩm.

Giao diện trang chủ ứng dụng phía máy chủ như Hình 13.

\section{* Thu thập dũ liệu phục vụ cho việc định hướng thông tin cho người dùng}

Có thể nói rằng lợi ích lớn nhất của việc sử dụng Beacon là khả năng thu thập đồng thời dữ liệu của người sử dụng cùng với dữ liệu dựa trên vị trí. Việc sử dụng Beacon trong các ứng dụng cho phép sử dụng công nghệ định vị trong nhà để chủ sở hữu ứng dụng có thể theo dõi dữ liệu hành vi của người sử dụng tại các cửa hàng hay sự kiện. Dữ liệu cá nhân của người sử dụng được thu thập thông qua ứng dụng có thể được người sử dụng nhập bằng tay hoặc qua hình thức xác thực thông qua tài khoản trên các dịch vụ xã hội - ví dụ thu thập dữ liệu về lối sống để đưa vào dữ liệu các hành vi của người dùng để tăng cường hiểu biết về tiếp thị. Các dữ liệu thu thập có thể bảo gồm tên, địa chỉ email, ngày sinh, số điện thoại, địa điểm, thành phố, mối quan hệ với các sản phẩm, ảnh hưởng của mạng xã hội và nhân khẩu học, tùy thuộc vào mục tiêu của từng ứng dụng. Bản thân các Beacon chỉ thu thập dữ liệu vị trí của người dùng. Bằng cách này dữ liệu thu thập qua ứng dụng được tăng theo cấp số nhân.
Với việc thu thập các dũ̃ liệu phù hợp thông qua ứng dụng di động, chủ sở hữu ứng dụng có thể tận dụng các mối quan hệ thương hiệu khách hàng và nhân khẩu học để thu hút các nhà tài trợ, cung cấp trải nghiệm cho khách hàng tốt hơn bằng cách đưa tới khách hàng các thông tin về sản phẩm như mô tả sản phẩm, giá cả, chương trình giảm giá, mã giảm giá đúng thời điểm, đúng đối tượng, qua đó làm tăng doanh số, tỉ lệ mua hàng.

\section{Kết luận}

Bài báo được thực hiện xây dựng một hệ thống IoT định hướng thông tin cho người dùng sử dụng công nghệ BLE. Thiết bị ESBeacon sử dụng công nghệ BLE được chế tạo thử nghiệm và đánh giá hiệu quả hoạt động so với thiết bị Beacon của hãng Estimote đang được bán trên thị thường Việt Nam. Hệ thống siêu thị thông minh ESMarket sử dụng ESBeacon được xây dựng với mục đích thử nghiệm và có thể được sử dụng trong các ứng dụng khác như văn phòng, sân bay, khách sạn, thư viện, bảo tàng,... Để thử nghiệm, các ESBeacon được đặt trong siêu thị và điện thoại thông minh hỗ trợ Bluetooth được sử dụng để cài đặt ứng dụng siêu thị kỹ thuật số để giao tiếp với ESBeacon. Kết quả cho thấy khi điện thoại thông minh xuất hiện trong phạm vi của ESBeacon, các thông tin liên quan về kệ hàng sẽ được hiển thị trên màn hình điện thoại.

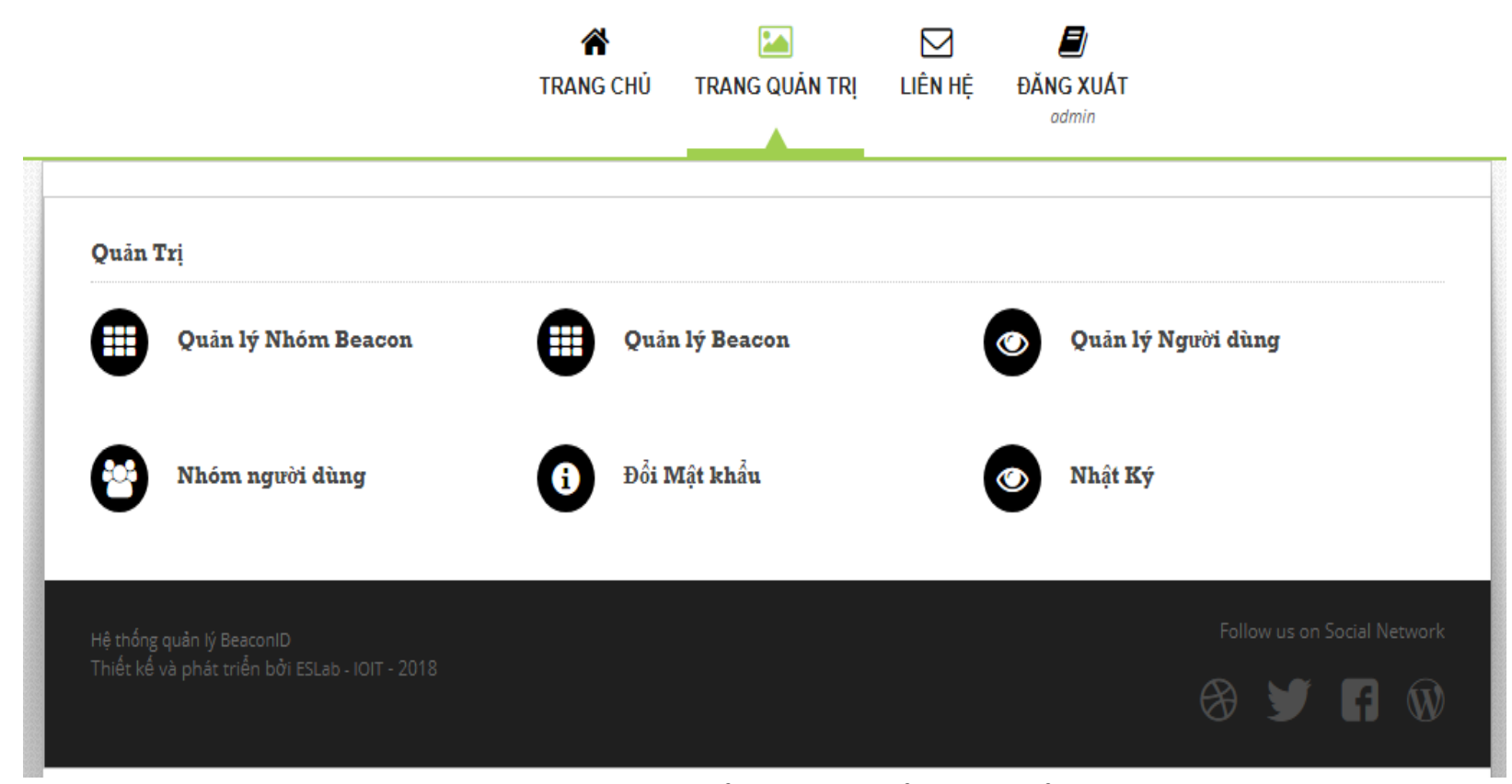

Hình 13. Trang chủ úng dụng của máy chủ. 


\section{Lò̀i cảm ơn}

Bài báo được hoàn thành với sự tài trợ của đề tài "Nghiên cứu và phát triển hệ thống IoT hỗ trợ" định hướng thông tin cho người dùng trên nền tảng điện toán đám mây", Viện Công nghệ thông tin - Viện Hàn lâm Khoa học và Công nghệ Việt Nam, mã số: VAST01.10/18 - 19.

\section{Tài liệu tham khảo}

https://altbeacon.github.io/android - beacon library/distance - calculations.html

Mohamad Omar Al Kalaa, Walid Balid, Naim Bitar, Hazem Refai, (2016). Evaluating Bluetooth Low Energy in Realistic Wireless
Environments. IEEE Wireless Communications and Networking Conference - Track 4 - Services, Applications, and Business, Doha, Quatar. https://www.researchgate.net /publication/291817370_Evaluating_Bluetoo th_Low_Energy_in_Realistic_Wireless_Environ ments.

Monika Adarsh, (2018). BLE beacon technology made simple: A complete guide to Bluetooth Low Energy Beacons, https://blog.beaconstac. com/2018/08/ble-made-simple-a-completeguide-to-ble-bluetooth-beacons /

Rappaport, T. S., (1996). Wireless Communications: Principles \& Practice, Prentice - Hall: Upper Saddle River, N. J. 